\title{
ASPECTOS GERAIS DA QUÍMICA DOS DITIOCARBAMATOS E DE SEUS COMPLEXOS METÁLICOS E INTERAÇÕES DESSAS ESPÉCIES QUÍMICAS COM IMPORTANTES ENZIMAS - UMA BREVE REVISÃO
}

\author{
Daniele C. Menezes ${ }^{\mathrm{a}}$ e Geraldo M. de Lima ${ }^{\mathrm{b}, *, \text { (i) }}$ \\ aDepartamento de Química, Universidade Federal de Viçosa, Viçosa - MG, Brasil \\ bDepartamento de Química, Universidade Federal de Minas Gerais, Belo Horizonte - MG, Brasil
}

Recebido em 06/12/2020; aceito em 25/03/2021; publicado na web em 22/04/2021

\begin{abstract}
GENERAL ASPECTS OF THE CHEMISTRY OF DITHIOCARBAMATES, THEIR METALLIC COMPLEXES AND THEIR INTERACTIONS WITH IMPORTANTE ENZYMES - A BRIEF REVISION. Dithiocarbamates (DTCs) comprise a series of compounds included in the class of 1,1 dithiolates. The application of these organic molecules in the coordination chemistry of some metals is well explored in the last decades, along with their potential applications in medicinal chemistry. In view of our expertise in this field, the syntheses of new DTCS, their coordination chemistry towards various metallic cations and their biocide activities, we summarize in this manuscript the outcomes of some investigations registered in the literature, involving the interactions of DTCs or their complexes with some key enzymes such as carbonic anhydrases, superoxide dismutase, urease, tyrosinase, glutathione, caspases and dehydrogenases. It is provided with a brief introduction of the chemistry of DTCs, electrochemistry, the kinetics of formation, solubility and stability towards solvation. The next topics summarize the results of some experiments concerning the inhibition of these cited enzymes effected by DTCs and some of their coordination complexes. The final remarks and conclusion of the outcomes are provided at the end of the manuscript.
\end{abstract}

Keywords: dithiocarbamates; dithiocarbamate complexes and inhibition of enzymes.

\section{INTRODUÇÃO}

A classe química dos compostos denominados ditiolatos incluem uma variedade de substâncias tais como os ditiocarbamatos (DTCs) $\mathrm{R}^{1} \mathrm{R}^{2} \mathrm{NCS}_{2}^{-}$(1), ditiofosfatos $\left(\mathrm{R}^{1} \mathrm{OR}^{2} \mathrm{OPS}_{2}^{-}\right)(2)$, disulfetos $\left(\mathrm{R}_{2} \mathrm{NSCS}_{-\mathrm{SCSNR}}\right)\left(\mathrm{R}^{1}\right.$ e $\mathrm{R}^{2}=$ grupos alguilas ou arilas, $\mathrm{R}^{1} \neq \mathrm{R}^{2} \mathrm{e}$ $\mathrm{R}^{1}$ ou $\mathrm{R}^{2}=\mathrm{H}$ ) (3) e xantatos $\left(\mathrm{ROCS}_{2}^{-}\right)$(4), Figura 1. Essas moléculas são importantes bases de Lewis com vasto potencial de aplicação em química de coordenação, capazes de estabilizar cátions metálicos nos mais variados estados de oxidação, formando complexos com uma grande diversidade de geometrias e propriedades.<smiles>[R]N([R])C(=S)[S-]</smiles>

(1)<smiles>[R]O[P+](=S)([S-])O[R]</smiles>

(2)<smiles>[R]N([R])C(=S)SSC(=S)N([R])[R]</smiles>

(3)<smiles>[R]OC(=S)[S-]</smiles>

(4)
Figura 1. Representação estrutural de alguns ditiolatos

Tendo em vista nossa experiência na química inorgânica dos DTCs e de seus complexos metálicos, suas propriedades químicas e aplicações biológicas, relatadas em nossos artigos publicados nas últimas duas décadas, reunimos nesse manuscrito informações relevantes sobre a atuação desses compostos na inibição de alguns processos enzimáticos. ${ }^{1-11}$ Dentre as diversas aplicações

\footnotetext{
*e-mail: delima.geraldo@gmail.com
}

dos ditiocarbamatos destacam-se seus empregos como pesticidas, catalisadores em alguns processos industriais, como por exemplo na vulzanização da borracha, além de possuírem potenciais aplicações como agentes antitumorais e biocidas. ${ }^{12-16}$ Alguns artigos relataram inclusive atividade anti-HIV. Antabuse (disulfiram), Figura 2, usado há muitos anos no tratamento de alcoolismo crônico, foi estudado como agente antitumoral. ${ }^{17-19}$

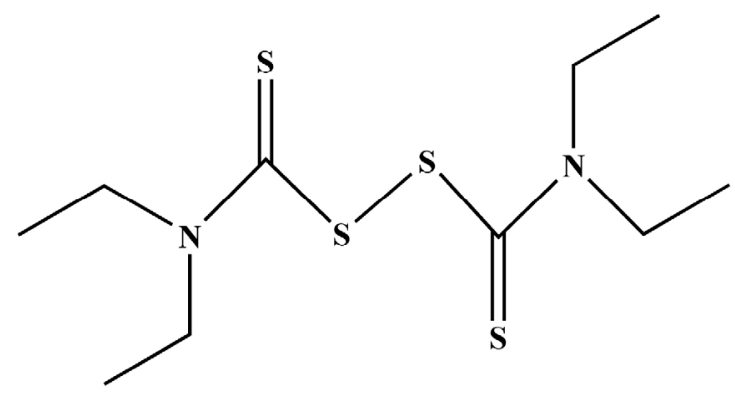

Figura 2. Representação estrutural do composto disulfiram

Essa pequena revisão reúne aspectos chaves para o entendimento de possíveis mecanismos de ação de ditiocarbamatos bem como de seus complexos metálicos. Não é pretensão dos autores esgotar nesse artigo o assunto por ser um tópico vasto e rico em detalhes, no entanto acreditamos que essas informações pontuadas aqui poderão ser utilizadas por especialistas na área de química inorgânica medicinal como ponto de partida para estudos mais pormenorizados. São considerados nesse manuscrito aspectos gerais da síntese dessas moléculas e de sua eletroquímica. Levam-se em consideração estudos sobre as constantes de formação de alguns complexos e sobre a estabilidade dos mesmos em meio aquoso. Finalmente, discutemse aspectos biológicos relacionados com a inibição enzimática efetuada por alguns DTCs, seus complexos e suas interações com biomoléculas. 


\section{ASPECTOS GERAIS SOBRE A OBTENÇÃO DE DITIOCARBAMATOS}

Os ditiocarbamatos (DTCs) são de fácil preparação, que se dá através da reação, em meio alcalino $(\mathrm{NaOH}$ ou $\mathrm{KOH})$, do $\mathrm{CS}_{2}$ com compostos orgânicos contendo pelo menos um grupo $\mathrm{R}_{1} \mathrm{R}_{2} \mathrm{~N}-\mathrm{H}$. Em geral emprega-se etanol, metanol ou mesmo água como solvente. Observa-se normalmente a precipitação imediata do ditiocarbamato do cátion do metal alcalino. Entretanto, isso depende da natureza dos grupos orgânicos presentes na estrutura. Após filtração o sólido é lavado com um solvente orgânico como tolueno e recristalizado numa mistura de solventes. Utiliza-se na recristalização um solvente volátil no qual o sal seja solúvel, pelo menos à quente, e tolueno ou n-heptano, menos volátil. É necessário ter cuidado para não ocorrer a precipitação do sal à medida que o segundo solvente é adicionado. A evaporação lenta do solvente mais volátil permitirá obter cristais do sal. Geralmente uma recristalização é suficiente para se obter uma estreita faixa de fusão $\left(<2^{\circ} \mathrm{C}\right)$. Esse sal é geralmente obtido com rendimento acima de $90 \%$, estável ao ar e solúvel em solventes próticos, o que facilita muito seu emprego em subsequentes reações com sais de cátions metálicos, Figura 3.

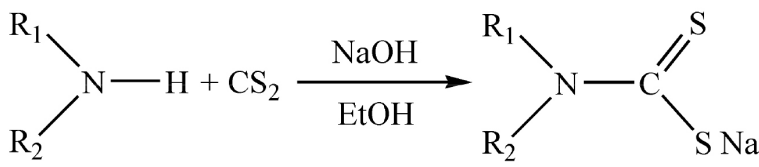

Figura 3. Esquema geral de obtenção de ditiocarbamatos

Ressalta-se que os ditiocarbamatos podem ser obtidos também na forma de sais de $\mathrm{NH}_{4}{ }^{+}$. Suas subsequentes reações com cátions de metais de transição e representativos utilizando-se diferentes estratégias sintéticas, levam à formação de complexos com os mais variados tipos de geometria. ${ }^{1-11}$ Esses complexos são inertes, estáveis e normalmente cristalinos, o que permite estudos por cristalografia de raios-X. É raro um complexo desse tipo que não seja solúvel, pelo menos em dimetilsulfóxido, facilitando a obtenção de informações preciosas através de experimentos de Ressonância Magnética Multinuclear. ${ }^{20-22}$

Os ligantes DTCs ligam-se a cátions metálicos preferencialmente de forma bidentada Figura 4 (a) ou monodentada (b). No primeiro caso ambos os enxofres são usados como centros doadores de elétrons, levando à formação de um anel quelato de quatro membros. Existem exemplos de complexos na literatura em que esses ligantes coordenam-se a mais de um centro metálico formando pontes entre os cátions metálicos Figura $4(\mathrm{c}-\mathrm{e})$.<smiles>CN(C)C1=NS1</smiles>

(a)<smiles>[M]SC(=S)N(C)C</smiles>

(b)

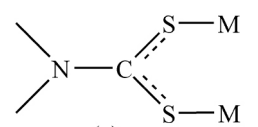

(c)

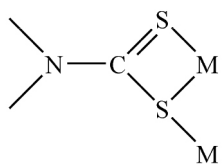

(d)

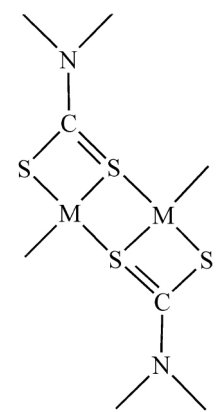

(e)

Figura 4. Representação dos diferentes modos de coordenação dos ligantes ditiocarbamatos aos centros metálico
As vibrações do grupo - $\mathrm{CS}_{2}$, estudadas através da espectroscopia no infravermelho, permitem um estudo detalhado do modo de coordenação do DTC ao centro metálico, bidentada ou monodentada. Se o ligante se liga de forma bidentada, duas situações podem ser detectadas no espectro vibracional. Pode-se observar apenas uma vibração $v_{\text {ass }}$ entre $1100-900 \mathrm{~cm}^{-1}$, ou então dois sinais bastante próximos $\left(\Delta v_{\text {ass }}<20 \mathrm{~cm}^{-1}\right)$ devido à pequena assimetria das ligações C-S. Essa assimetria aumenta quando o ligante está ligado ao cátion metálico de forma monodentada, $\mathrm{M}-\mathrm{S}-\mathrm{C}=\mathrm{S}\left(\Delta \mathrm{v}_{\text {ass }}>20 \mathrm{~cm}^{-1}\right){ }^{8}$

Estudos de $\mathrm{RMN}$ de ${ }^{13} \mathrm{C}$ de complexos diamagnéticos podem fornecer resultados muito interessantes. $\mathrm{O}$ sinal correspondente ao $\delta$ $\left({ }^{13} \mathrm{CS}_{2}\right)$ do ligante desloca-se para frequências menores nos complexos, indicando um maior efeito de blindagem devido ao um possível aumento da densidade eletrônica em torno do átomo de carbono. De fato, a ligação entre $\mathrm{N}$ e $\mathrm{C}$ tem maior caráter de dupla nos complexos, tendo em vista o aumento da frequência de estiramento N-C no grupo $\left(\mathrm{NCS}_{2}\right)$ observado no infravermelho. ${ }^{23}$ É bom salientar a importância desses estudos correlacionando resultados de diferentes técnicas de caracterização na formação de estudantes de pós-graduação.

\section{ASPECTOS GERAIS DA ELETROQUÍMICA DOS DITIOCARBAMATOS (DTCS)}

Apesar da estabilidade química da maioria dos complexos contendo DTC como ligantes, alguns compostos com metais pesados exibindo alto estados de oxidação, podem ser reduzidos, causando a oxidação do grupo DTC, formando disulfetos. Dessa forma, o complexo perde um ou mais ligantes, levando à formação de sítios vagos de coordenação. Nessa forma reduzida esses complexos podem exibir um grande potencial para respostas biológicas, por possuírem um certo grau de biodisponibilidade frente a uma variedade de bio-moléculas. Como já mencionado, ditiocarbamatos coordenam metais em diferentes estados de oxidação, podendo exibir padrões eletroquímicos ricos em informações importantes e variados tipos de reações redox. Complexos dos cátions $\mathrm{Cu}(\mathrm{I}), \mathrm{Ni}(\mathrm{II}), \mathrm{Zn}(\mathrm{II}), \mathrm{Cd}(\mathrm{II})$ e $\mathrm{Hg}$ (II) coordenados ao grupo dibutilditiocarbamato, $\mathrm{bu}_{2} \mathrm{dtc}^{2-}$, foram relatados em um trabalho publicado no final da década de $1960 .{ }^{24}$ Esses complexos foram oxidados na presença de diferentes agentes oxidantes. A forma oxidada do complexo de níquel apresentou uma composição [ $\mathrm{Ni}\left(\mathrm{bu}_{2} \mathrm{dtc}\right)_{3}$ ], enquanto a caracterização dos outros complexos levou à proposta das seguintes formulações: $\left[\mathrm{Cu}\left(\mathrm{bu}_{2} \mathrm{dtc}\right) \mathrm{X}_{3}\right]\left(\mathrm{X}=\mathrm{Cl}, \mathrm{Br}, \mathrm{NO}_{3}\right), \mathrm{M}\left(\mathrm{bu}_{2} \mathrm{dtc}_{2} \mathrm{X}_{2}(\mathrm{M}=\mathrm{Zn}, \mathrm{Cd}\right.$ e Hg; $\mathrm{X}=\mathrm{Br}$ ou I). Nesse trabalho relatam-se as seguintes oxirreduções dos centros metálicos $\mathrm{Ni}(\mathrm{II}) / \mathrm{Ni}(\mathrm{IV}), \mathrm{Cu}(\mathrm{I}) / \mathrm{Cu}(\mathrm{IV})$ e $\mathrm{M}(\mathrm{II}) / \mathrm{M}(\mathrm{IV})(\mathrm{M}=\mathrm{Zn}$, $\mathrm{Cd}$ e $\mathrm{Hg}$ ). Embora as análises elementares dos produtos da oxidação $\mathrm{M}(\mathrm{II}) / \mathrm{M}(\mathrm{IV})$ sugerem estados de oxidação incomuns dos cátions do grupo 12, a ausência de outras evidências torna inconclusiva a formulação proposta, $\mathrm{M}\left(\mathrm{bu}_{2} \mathrm{dtc}\right)_{2} \mathrm{X}_{2}(\mathrm{M}=\mathrm{Zn}, \mathrm{Cd}$ e $\mathrm{Hg} ; \mathrm{X}=\mathrm{Br}$ ou I $){ }^{24}$

Estudos envolvendo reações redox de complexos de cobalto $\left[\mathrm{Co}\left(\mathrm{S}_{2} \mathrm{CNR}_{2}\right)_{2}\right](\mathrm{R}=\mathrm{N}$-metilfenil, 1-fenilpiperazil, morfolinil)] foram feitos em 2019. O processo de recristalização dos complexos em diclorometano leva a oxidação espontânea do Co(II) para Co(III). Os cristais isolados na verdade exibem três ligantes para cada cátion cobalto, $\left[\mathrm{Co}\left(\mathrm{S}_{2} \mathrm{CNR}_{2}\right)_{3}\right]$. Estudos de voltametria cíclica revelaram um processo redox quase reversível $\mathrm{Co}(\mathrm{III})->\mathrm{Co}(\mathrm{IV})->\mathrm{Co}(\mathrm{III}) .{ }^{25}$

\section{CINÉTICA E TERMODINÂMICA DE FORMAÇÃO DE ALGUNS COMPLEXOS CONTENDO DITIOCARBAMATOS COMO LIGANTES}

Ainda são escassas as informações na literatura sobre a cinética de formação de complexos contendo ditiocarbamatos. Um dos primeiros trabalhos documentados na literatura relata a avaliação das 
constantes de formação de complexos do tipo $\left[\mathrm{M}\left(\mathrm{S}_{2} \mathrm{CNR}_{2}\right)_{2}\right]\{\mathrm{M}=$ $\mathrm{Cu}(\mathrm{II}), \mathrm{Ni}(\mathrm{II}), \mathrm{Cd}(\mathrm{II})$ e $\mathrm{Zn}(\mathrm{II})\} .{ }^{26} \mathrm{O}$ maior valor de $\mathrm{K}$ foi obtido para o derivado contendo $\mathrm{Cu}$ (II). Observa-se que a natureza do grupo $\mathrm{R}$ tem importância fundamental na estabilidade desses compostos, por exemplo, $\left[\mathrm{Ni}\left(\mathrm{S}_{2} \mathrm{CNR}_{2}\right)_{2}\right]$ contendo o grupo $\mathrm{R}=$ pirrolidina é menos estável que os derivados onde $\mathrm{R}=$ metil, etil, propil ou butil. $\mathrm{O}$ complexo contendo pirrolidina confere maior labilidade ao complexo de $\mathrm{Ni}(\mathrm{II})$, tornando-se adequado para investigações de suas atividades biológicas. $^{26}$

A reação entre $\left[\mathrm{Cu}(\mathrm{dtc})_{2}\right]$ com $\mathrm{NO}$ e meio aquoso foi estudada posteriormente, Figura 5. As constantes de formação bem como os diagramas de especiação para os produtos dessa reação, $\left[\mathrm{Cu}(\mathrm{dtc})_{2} \mathrm{NO}\right]$ e $\left[\mathrm{Cu}(\mathrm{dtc})_{2}(\mathrm{NO})_{2}\right]$, foram determinados. ${ }^{27}$

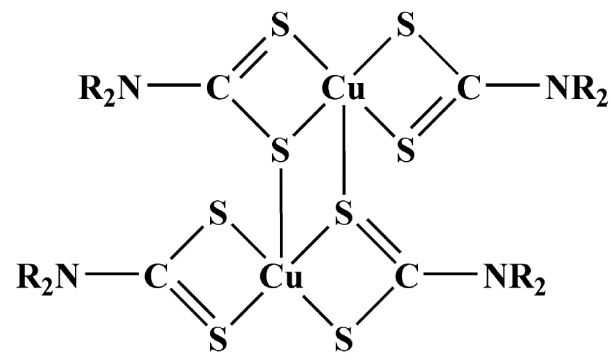
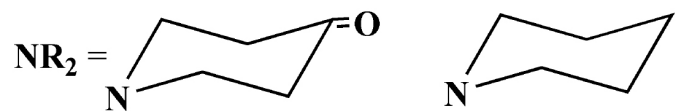

Figura 5. Representação estrutural do complexo $\left[\mathrm{Cu}(\mathrm{dtc})_{2}\right]$

Os resultados permitiram concluir que a formação do complexo $\left[\mathrm{Cu}(\mathrm{dtc})_{2} \mathrm{NO}\right]$ na proporção de $(1: 1)$ é termodinamicamente favorecida, em relação ao $\left[\mathrm{Cu}(\mathrm{dtc})_{2}(\mathrm{NO})_{2}\right]$, que tem uma cinética mais rápida de formação, por exibir maior valor de $\mathrm{k}_{1}$. Os dois complexos, $\left[\mathrm{Cu}(\mathrm{dtc})_{2} \mathrm{NO}\right]$ e $\left[\mathrm{Cu}(\mathrm{dtc})_{2}(\mathrm{NO})_{2}\right]$, se interconvertem através de uma reação reversível:

$$
\left[\mathrm{Cu}(\mathrm{dtc})_{2}: \mathrm{NO}\right]+\mathrm{NO} \leftrightarrows\left[\mathrm{Cu}(\mathrm{dtc})_{2}(\mathrm{NO})_{2}\right]
$$

Segundo os autores, todos as reações ocorrem de maneira reversível e os valores de constantes de formação obtidos $\left(10,3 \geq \log \mathrm{K}_{1} \geq 3,16 ; 3,9 \geq \log \mathrm{K}_{2} \geq 2,2\right)$ revelam que o esse complexo se apresenta como possível candidato na captura de óxido nítrico em condições fisiológicas com consequências biológicas relevantes.

As constantes de formação de complexos de $\mathrm{Cu}(\mathrm{II})$ de fórmula geral $\left[\left(\mathrm{R}^{1}\left(\mathrm{CH}_{2} \mathrm{CH}_{2} \mathrm{O}\right)_{2} \mathrm{NR}^{2} \mathrm{CS}_{2}\right)_{2} \mathrm{Cu}\right]$ (com $\mathrm{R}^{1}=$ etil, butil, hexil; $\mathrm{R}^{2}=$ etil) foram estimadas com o emprego de um método de competição dos ligantes ditiocarbamatos e 8-hidroxiquinolina frente a íons $\mathrm{Cu}^{2+}$. Os elevados valores obtidos de $\mathrm{K}_{\mathrm{c}}$ para os ligantes ditiocarbamatos em questão $\left(\mathrm{K}_{\mathrm{c}}=0,34 \times 10^{2} ; 0,89 \times 10^{2}\right.$ e $1,07 \times 10^{2}$ para ditiocarbamatos com $\mathrm{R}^{1}=$ etil, butil e hexil, respectivamente) demonstram uma forte afinidade por íons $\mathrm{Cu}^{2+}$ em relação a 8-hidroxiquinolina, explicitando que estes derivados são bons agentes complexantes frente a este centro metálico. ${ }^{28}$

Em um trabalho de 2018 determinou-se o mecanismo e a cinética de formação do complexo $\left[\mathrm{Cu}\left(\mathrm{S}_{2} \mathrm{CNBu}_{2}\right)_{2}\right]$, Figura $6 .^{29}$

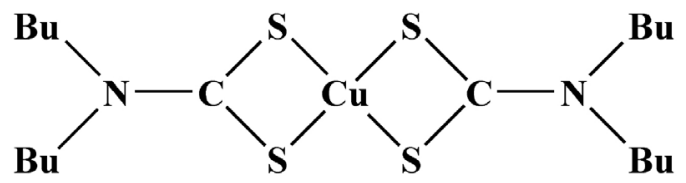

Figura 6. Representação estrutural do complexo $\left[\mathrm{Cu}\left(\mathrm{S}_{2} \mathrm{CNBu}_{2}\right)_{2}\right]$
Os autores estudaram a cinética e o tempo de formação do complexo em questão, em função do $\mathrm{pH}$ e da concentração do DTC usado como ligante. Determinou-se que a velocidade de formação do complexo segue a expressão: $\mathrm{V}=0,917106 \mathrm{x}\left[\mathrm{Cu}(\mathrm{II}]^{0,87921} \times\left[\mathrm{S}_{2} \mathrm{CNBu}_{2}\right]^{2,03021}\right.$ Observou-se que as melhores condições de reação envolvem um $\mathrm{pH}=3$ e $[\mathrm{DTC}]=4 \times 10^{-3} \mathrm{~mol} \mathrm{~L}^{-1}$. Os resultados obtidos nesse trabalho sugerem que a formação do complexo ocorre em duas etapas. A primeira etapa envolve a formação da espécie $\left[\mathrm{Cu}\left(\mathrm{S}_{2} \mathrm{CNBu}_{2}\right)\right]^{+}$, enquanto na segunda etapa outro ligante é adicionado à esfera de coordenação do $\mathrm{Cu}(\mathrm{II})$, formando então $\left[\mathrm{Cu}\left(\mathrm{S}_{2} \mathrm{CNBu}_{2}\right)_{2}\right]{ }^{29}$

\section{SOLUBILIDADE DE ALGUNS DTCS E DE SEUS COMPLEXOS}

O coeficiente de partição na mistura octanol/água $\left(\mathrm{P}_{\mathrm{ow}}\right)$ é frequentemente utilizado para estudar o transporte de substâncias em barreiras hidrofóbicas, como por exemplo a membrana celular. Dezoito ditiocarbamatos com diferentes grupos $\mathrm{R}$ foram avaliados através de modelagem molecular e estudos de QSAR. Nesse estudo, o DTC contendo o grupo pirrolidino mostrou o menor valor de $\mathrm{P}_{\mathrm{ow}} \mathrm{e}$ uma maior polaridade em comparação a outros derivados contendo grupos metil, etil, propil ou butil. ${ }^{30}$

Um estudo recente envolvendo o complexo $\left[\mathrm{Au}\left\{\mathrm{S}_{2} \mathrm{CN}(\mathrm{Me})\right.\right.$ $\left.\left(\mathrm{CH}_{2} \mathrm{COOCH}_{2} \mathrm{CH}_{3}\right)\right\} \mathrm{Br}_{2}$ ], Figura 7 , mostrou um aumento de sua solubilidade e estabilidade química ao ser encapsulado por $\beta$-cyclodextrina. ${ }^{31}$

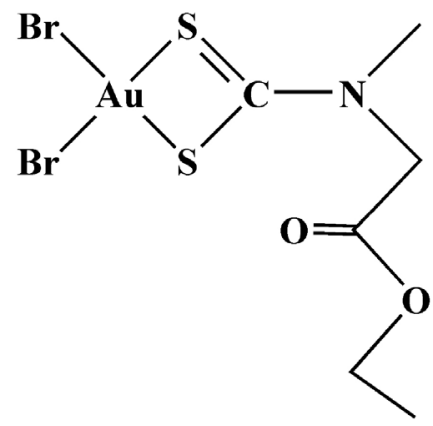

Figura 7. Representação estrutural do complexo $\left[\mathrm{Au}\left\{\mathrm{S}_{2} \mathrm{CN}(\mathrm{Me})\left(\mathrm{CH}_{2} \mathrm{COO}\right.\right.\right.$ $\left.\left.\left.\mathrm{CH}_{2} \mathrm{CH}_{3}\right)\right\} \mathrm{Br}_{2}\right]$

A atividade antitumoral do material resultante foi testada na presença de algumas linhagens de células, por exemplo, HeLa, KB e MCF7, mostrando excelentes resultados antitumorais e baixa toxicidade. Esse complexo encapsulado mostrou-se mais biodisponível, mais estável e mais solúvel em água.

Um procedimento similar utilizando o polímero Pluronic F127 foi empregado no encapsulamento de complexos mononucleares e dinucleares, produzidos pela reação de $\mathrm{Ru}(\mathrm{III})$ com ditiocarbamatos contendo os grupos pirrolidino ou carbazol, Figura $8 \mathrm{a}$ e 8 b, respectivamente..$^{32}$
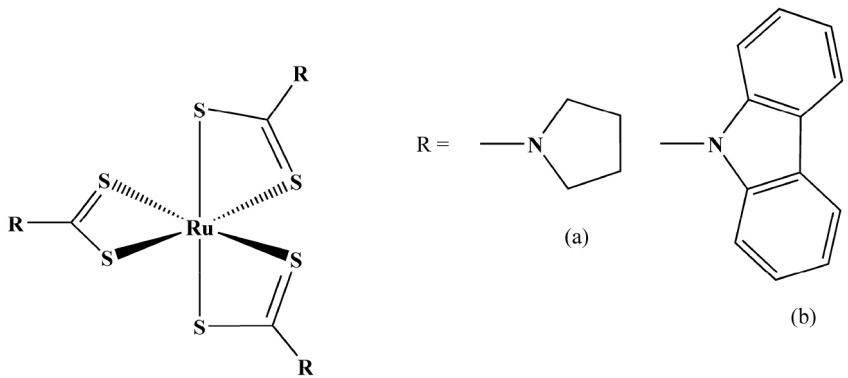

(b)

Figura 8. Representação estrutural do complexo $\left[\mathrm{Ru}\left(\mathrm{S}_{2} \mathrm{CNR}_{2}\right)_{3}\right]$ 


\section{ESTABILIDADE DE DTCS FRENTE A HIDRÓLISE}

A decomposição de DTCs, produzindo aminas e dissulfeto de carbono, mostrou-se dependente de pH. Estudou-se a hidrólise em meio alcalino dos compostos representados por $\mathrm{R}^{\prime}\left(\mathrm{CH}_{2} \mathrm{CH}_{2} \mathrm{O}\right)_{2} \mathrm{NR}^{\prime} \mathrm{CS}_{2} \mathrm{Na}\left\{\mathrm{R}^{\prime}=\right.$ etil, butil, hexil, octil, decil ou dodecil; $\mathrm{R}^{\prime}=$ etil e butil $\}$. Observou-se que os derivados contendo os grupos decil e dodecil apresentam maior suscetibilidade a sofrer hidrólise. ${ }^{28}$

\section{INIBIÇÃO DE IMPORTANTES PROCESSOS ENZIMÁTICOS POR DTCS}

\section{Anidrases carbônicas}

Anidrases carbônicas formam uma família de proteínas e enzimas contendo $\mathrm{Zn}$ (II) que catalisam importantes processos biológicos em vertebrados superiores, bactérias, algas e plantas. Em mamíferos, essas substâncias ajudam a manter processos bioquímicos, tais como o equilíbrio ácido-base do sangue e de outros tecidos, bem

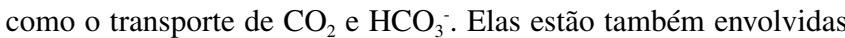
na conversão de cianatos em ácido carbâmico, na síntese da ureia e no desenvolvimento de processos patológicos, como glaucoma e tumores malignos. Diante disso, é de se supor que essa enzima pode ser um bom alvo no estudo de novas terapias para o controle de determinadas moléstias. ${ }^{33}$

A estrutura cristalográfica da anidrase carbônica exibe $\mathrm{Zn}$ (II) como centro metálico, coordenado por três fragmentos de histidina (His 94, His 96 e His 119). A quarta posição de coordenação pode alojar uma molécula de água ou $\mathrm{OH}^{-}$. A forma ativa da enzima possui uma hidroxila coordenando o cátion $\mathrm{Zn}(\mathrm{II})$, responsável pela conversão de $\mathrm{CO}_{2}$ em $\mathrm{HCO}_{3}{ }^{-}$. Após esse processo, uma molécula de água ocupa essa posição, tornando a enzima inativa. A transferência de $\mathrm{H}^{+}$para um fragmento de aminoácido, considerada a etapa cinética limitante, ativa a enzima novamente. ${ }^{34}$

Sulfonamidas na forma aniônica são conhecidos inibidores de anidrases carbônicas. Essas substâncias coordenam-se ao centro metálico, Zn(II), levando à formação de estruturas tetraédricas ou trigonal bipiramidais. Drogas que exibem sulfonamidas como princípio ativos podem ser utilizadas no tratamento de glaucoma, inibindo a ação de anidrases carbônicas presentes no olho, permitindo a redução de íons bicarbonatos no líquido ocular. ${ }^{35}$

Supuran e seu grupo de pesquisa investigaram não só a inibição dessas enzimas usando ditiocarbamatos mas também o potencial dos DTCs na terapia de infecções causadas por fungos. Os DTCs utilizados apresentaram os grupos piperidina, morfolina and piperazina em suas estruturas. Alguns deles apresentaram excelente resultados comparados com acetazolamida, usada como droga controle.$^{36}$ Esse mesmo grupo relatou resultados sobre a eficácia de DTCs e do ânion tritiocarbonato, $\mathrm{CS}_{3}{ }^{-}$, em comparação com o efeito de outros ânions $\mathrm{SnO}_{3}{ }^{2-}, \mathrm{SeO}_{4}{ }^{2-}$ e $\mathrm{TeO}_{4}{ }^{2-} \cdot{ }^{37}$

As estruturas cristalográficas obtidas para os adutos formados entre três ditiocarbamatos e anidrase carbônica humana foram elucidadas por difração de raios-X. Esse experimento revelou que apenas um dos átomos de enxofre se liga ao $\mathrm{Zn}$ (II), de forma monodentada. Esse cátion se encontra coordenado a fragmentos de aminoácidos, His94, His96 e His119 e ao ditiocarbamato, formando um arranjo geométrico tetraédrico. ${ }^{38}$ Além desse estudo, a literatura mostra vários trabalhos relacionados à inibição de anidrases carbônicas por ditiocarbamatos, demonstrando o potencial dessas moléculas de serem usadas no tratamento do glaucoma e de outras doenças causadas por bactérias patogênicas. ${ }^{39-41}$ Alguns trabalhos interessantes correlacionam a presença de anidrases carbônicas com a existência de tumores malignos, tornando essas enzimas possíveis alvos para o desenvolvimento de novas drogas antitumorais. ${ }^{42,43}$

\section{Superóxido dismutase (SOD)}

A enzima superóxido dismutase é responsável pela conversão catalítica do ânion superóxido, $\mathrm{O}_{2}^{-}$, em oxigênio e peróxido de hidrogênio. Os sítios metálicos $\mathrm{Cu}$ (II), Zn(II), Fe(III), Mn(II) e Ni(II) podem estar presentes nessas espécies químicas. Quando ocorre o chamado estresse oxidativo, o ânion superóxido é convertido em $\mathrm{H}_{2} \mathrm{O}_{2}$, o qual é mais tarde degradado na presença de glutationa peroxidase e catalase. Os cátions metálicos estão normalmente coordenados de forma lateral a três cadeias de histidina, um aspartato, uma molécula de água ou a um grupo hidroxila, dependendo de seus estados de oxidação. ${ }^{44}$

O estudo de inibidores dessa enzima é de grande importância ambiental, pois a ação dos mesmos representaria uma grande diminuição de produtos agroquímicos, estes associados a formulações contendo inibidores da superóxido dismutase. ${ }^{45}$

É sabido que excesso de superóxido dismutase pode levar ao desenvolvimento de doenças neurodegenerativas ou induzir a morte devido a presença de grandes concentrações de $\mathrm{H}_{2} \mathrm{O}_{2}$ no organismo. ${ }^{46-48} \mathrm{~A}$ literatura registra alguns estudos da inibição dessa enzima por ditiocarbamatos. Morfolinoditiocarbamato (MDTC) e dietilditiocarbamato (EDTC) inibem a oxidação do pirogalol, usado como molécula modelo, mostrando propriedades antioxidantes superiores ao ácido ascórbico. ${ }^{49}$ Os complexos metálicos resultantes da coordenação desses ligantes com $\mathrm{Cu}$ (II), $\mathrm{Co}$ (II) e $\mathrm{Ni}$ (II) foram mais efetivos na conversão do ânion $\mathrm{O}_{2}^{-}$. Os complexos $\left[\mathrm{Cu}(\mathrm{GluDTC})_{2}\right]$ e $\left[\mathrm{Cu}(\mathrm{GlyDTC})_{2}\right]$ (ditiocarbamatos derivados da glicina e do ácido glutâmico) exibiram pronunciada atividade antioxidante na presença do pirogalol. ${ }^{50}$

Reagiu-se o composto etilfenilditiocarbamato de sódio com $\mathrm{Co}(\mathrm{II}), \mathrm{Cu}(\mathrm{II}), \mathrm{Mn}(\mathrm{II}), \mathrm{Cr}(\mathrm{III})$ e $\mathrm{Pd}(\mathrm{II})$ produzindo os respectivos complexos. As atividades antimicrobiais desses complexos foram avaliadas na presença dos patógenos Escherichia coli, Pseudomonas aureginosa, Salmonella typhi, Staphylococcus aureus, Aspergillus flavus e Fusarium oxysporum. O complexo de $\mathrm{Cu}(\mathrm{II})$ e o ligante apresentaram propriedades antioxidades na presença de 2,2-difenil1-picril-hidrazil (DPPH). ${ }^{51}$

Alguns trabalhos publicados em 1992 relatam o uso de thiram, a forma oxidada do dímero dimetilditiocarbamato, Figura 9, como pesticida em sementes. Foram feitos experimentos para estudar o potencial mutagênico dessa molécula utilizando tecidos de fígado de peixes. ${ }^{52}$

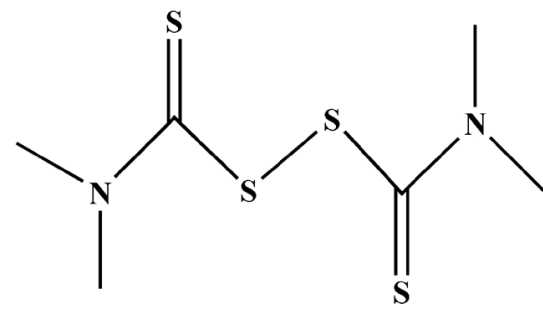

Figura 9. Representação estrutural do composto thiram

Os resultados desses experimentos foram bem ricos em informações, pois revelaram que a atividade antifúngica dessa molécula envolve a inibição das enzimas superóxido dismutase, catalase e glutationa peroxidase. ${ }^{52}$

A atividade enzimática do $\mathrm{Cu}-\mathrm{Zn}-\mathrm{SOD}$ foi estudada na presença do composto $\mathrm{R}^{1}\left(\mathrm{CH}_{2} \mathrm{CH}_{2} \mathrm{O}\right)_{2} \mathrm{NR}^{2} \mathrm{CS}_{2} \mathrm{Na}\left(\mathrm{R}^{1}=\right.$ etil, butil, hexil, octil, decil ou dodecil; $\mathrm{R}^{2}=$ etil e butil). Os experimentos foram monitorados 
por ressonância paramagnética eletrônica (EPR) ${ }^{53}$ Concluiu-se que os derivados contendo os grupos hexil e octil mostraram maior inibição da atividade enzimática.

\section{Urease}

Urease contém dois sítios catalíticos de $\mathrm{Ni}(\mathrm{II})$ e encontra-se presente em várias espécies de plantas, bactérias, fungos e algas..$^{54,55}$ Essa enzima é responsável pela transformação da ureia em amônia, sendo imprescindível em processos de fixação do $\mathrm{N}_{2}$ e no metabolismo dessa molécula pelas plantas. Na medicina, essa enzima está associada ao desenvolvimento de certos tipos de tumores malignos do trato digestivo e em processos infecciosos causados por alguns microorganismos Helicobacter pylori, Mycobacterium. tuberculosis, Cryptococcus neoformans ou por Penicillium spp.

Embora tenha sido cristalizada em 1926 por J. B. Sumner, sua estrutura só foi elucidada em 1995 por P. A. Karplus. ${ }^{56}$ Urease extraída de Klebsiella aerogenes revela uma estrutura trimérica contendo três sítios dinucleares de $\mathrm{Ni}(\mathrm{II}) \cdot{ }^{57} \mathrm{Em}$ sua forma ativa essa enzima possui um grupo carbamato, resultante da reação de $\mathrm{CO}_{2}$ com fragmentos de amino ácidos. Além dessas características a urease possui em sua estrutura vários grupos tióis, oriundos de doze fragmentos de cisteína. Um dos cations Ni(II) está cercado por dois grupos de histidina e uma molécula de água. O outro cátion $\mathrm{Ni}$ (II) coordena-se a dois fragmentos de histidina, uma molécula de água e um grupo aspartato. Os grupos tióis são normalmente os alvos em estudo de inibição dessa enzima. ${ }^{58-60}$

A molécula de $\mathrm{CO}_{2}$ pode ser substituída por $\mathrm{CS}_{2}$, formando ditiocarbamato e desativando a enzima. ${ }^{59}$ Sulforafano, $\mathrm{CH}_{3} \mathrm{~S}(=\mathrm{O})$ $\left(\mathrm{CH}_{2}\right)_{4} \mathrm{NCS}$, e outros isotiocianatos são fortes inibidores da urease produzida por $H$. pylori ${ }^{61}$ Mais recentemente, observou-se que o composto $\left[\mathrm{Bi}\left\{\mathrm{S}_{2} \mathrm{CN}\left(\mathrm{CH}_{2} \mathrm{CH}_{2} \mathrm{OH}\right)\left(\mathrm{CH}_{2} \mathrm{CH}_{2} \mathrm{CH}_{3}\right)\right\}_{3}\right]$, Figura 10, é um potente inibidor dessa enzima. ${ }^{62}$

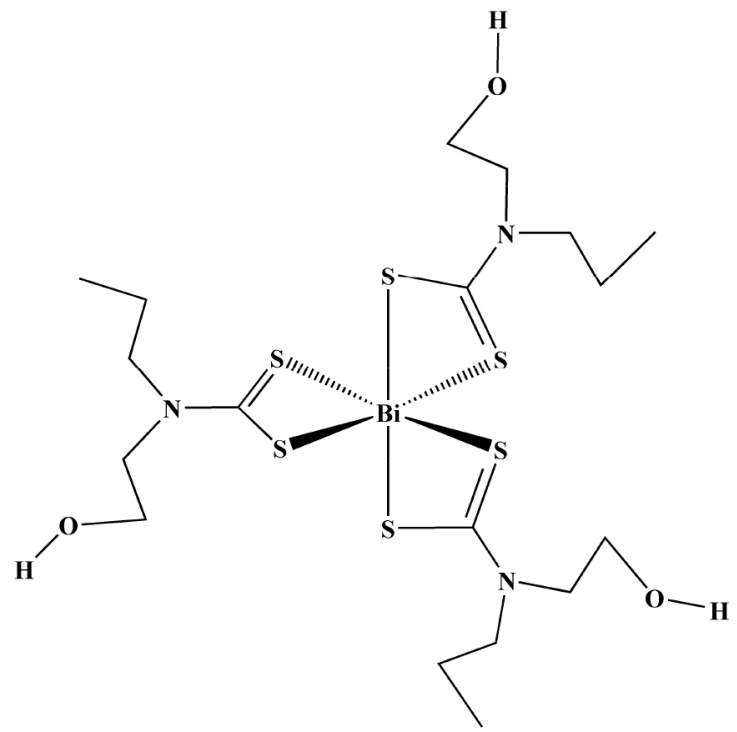

Figura 10. Representação estrutural do complexo $\left[\mathrm{Bi}\left\{\mathrm{S}_{2} \mathrm{CN}\left(\mathrm{CH}_{2} \mathrm{CH}_{2} \mathrm{OH}\right)\right.\right.$ $\left.\left.\left(\mathrm{CH}_{2} \mathrm{CH}_{2} \mathrm{CH}_{3}\right)\right\}_{3}\right]$

Nesse trabalho foram relatados vários parâmetros físico-químicos, tais como dados cinéticos e termodinâmicos, constante de velocidade, tempo de relaxação e energia livre de Gibbs. ${ }^{62} \mathrm{O}$ mecanismo proposto nesse trabalho envolve uma reação elementar de segunda ordem com dois estados de transição com estabilidade semelhante. Devido à biodisponibilidade do $\mathrm{Bi}$ (III) e sua afinidade por enxofre, é razoável supor que ocorre uma forte interação com grupos tióis, causando a desativação da enzima.

\section{Tirosinase}

A enzima tirosinase possui como sítios catalíticos cátions $\mathrm{Cu}$ (II) coordenados a fragmentos de histidina ${ }^{63}$ Essa enzima catalisa a hidroxilacão de fenóis a acetóis e subsequente oxidação a quininas. Essa última molécula é bastante reativa e pode polimerizar-se formando melanina, o bio-polímero responsável pelo excesso de pigmentação de vários vegetais e mamíferos. A tirosinase presente em cogumelos pode ser inibida na presença de $\mathrm{RNHCS}_{2} \mathrm{Na}$ contendo piperidina ou piperazina como grupos $\mathrm{R} .{ }^{64} \mathrm{Um}$ trabalho de revisão foi publicado em 2019, no qual os autores relatam aspectos importantes da inibição dessa enzima. ${ }^{65}$

\section{Glutationa}

Glutationa é um forte agente redutor que apresenta importante ação antioxidante no meio biológico. Essa enzima é um peptídeo formado por três aminoácidos: ácido glutâmico, cisteína e glicina. O grupo tiol da cisteína desempenha papel vital na atividade dessa enzima, pois em sua forma oxidada-SH perde o hidrogênio formando ligações -S-S-. Nesse caso a enzima é representada por GSSG. Já em sua forma reduzida, ou seja na forma de tiol, a representação é GSH. ${ }^{66}$ A sobrevivência da célula depende do equilíbrio GSH/GSSG. Nesse contexto duas classes de enzimas são importantes, a glutationa redutase $(\mathrm{GR})$ e a glutationa peroxidase $(\mathrm{GPx})$, parcialmente responsáveis por possíveis situações de estresse celular. ${ }^{67,68} \mathrm{O}$ mecanismo de algumas drogas para o tratamento de malária ou de infecções causadas por fungos tem como alvo o equilíbrio GR/ GPx. ${ }^{69,70}$

Alguns complexos de $\mathrm{Au}(\mathrm{III})$ contendo ditiocarbamatos como ligantes, Figura 11, exibiram excelentes resultados na presença de algumas células de tumores malignos, por exemplo: câncer de pulmão (A549), câncer de seio (MCF-7 e MDA-MB-231), câncer de colo do útero (HCT-116) e leucemia (H160). Resultados de RMN de ${ }^{1} \mathrm{H}$ e de UV/Vis sugerem que que um dos complexos é reduzido por glutationa, mas inativo frente a $\mathrm{N}$-acetil cisteína. ${ }^{7}$

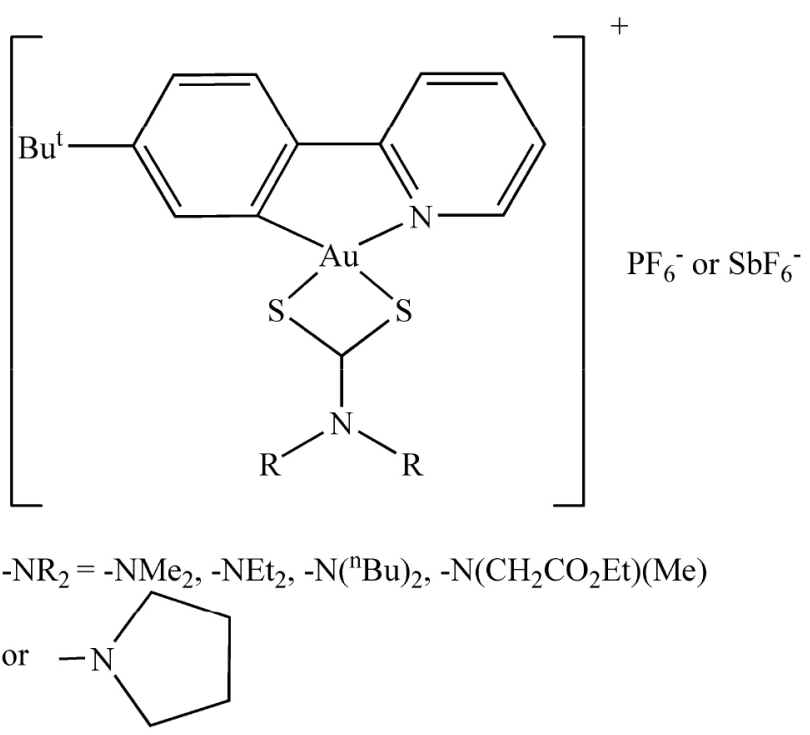

Figura 11. Representação estrutural de alguns ditiocarbamatos de $\mathrm{Au}(\mathrm{III})$

Comportamento similar de alguns ditiocarbamatos de $\mathrm{Au}(\mathrm{III})$ foram observados por Fregona e co-autores. ${ }^{72}$ Complexos com notada atividade antitumoral foram reduzidos pela glutationa (GSH), em experimentos monitorados por espectroscopia eletrônica e por RMN de ${ }^{1} \mathrm{H}$. Os resultados de RMN de ${ }^{1} \mathrm{H}$ mostraram a formação da 
espécie dimérica de $\mathrm{Au}(\mathrm{I})\left[\mathrm{Au}_{2}(\mathrm{dtc})_{2}\right]$ e de GSSG, a forma oxidada da glutationa. A espectroscopia eletrônica mostrou drástica redução das concentrações de GSH e do complexo durante a reação. ${ }^{72}$

Experimentos com ratos diabéticos demonstraram um aumento na produção de insulina e a consequente diminuição do nível glicêmico induzido por um ditiocarbamato contendo o grupo pirrolidino. Observou-se ainda que foram inibidas as atividades das enzimas superóxido dismutase e da glutationa peroxidase. ${ }^{73}$

Trabalhos de 20 ou 30 anos atrás já apontavam a diminuição das concentrações de GSH por ditiocarbamatos. ${ }^{74,75}$ Alguns artigos mostraram que células expostas a etilditiocarbamato (EDTC) podem ter seus níveis de GR aumentados e os de GPx diminuídos e que EDTC pode mimetizar glutationa peroxidases, utilizando glutationa como um substrato para a redução de peróxido de hidrogênio e hidroperóxidos orgânicos. ${ }^{76,77}$

Acredita-se que a glutationa possui um papel importante em processos de resistência ao tratamento de certos tipos de cânceres com compostos de platina. Células tumorais resistentes à cisplatina apresentam alto teor de glutationa, sendo que derivados do tipo $\left[\mathrm{Pt}(\mathrm{GS})_{2}\right]$ são formados e eliminados do organismo, reduzindo assim a ação quimioterápica desse importante fármaco. ${ }^{78}$

\section{Caspases}

Caspases constituem um grupo de proteases ricas em cisteína, responsáveis pela clivagem de outras proteínas especificamente após um resíduo de ácido aspártico, sendo sua denominação oriunda dessa função característica, cysteine-aspartic-acid-proteases. Caspases são de fundamental importância no processo de apoptose celular. Falhas na apoptose poderão ocasionar o desenvolvimento de tumores, doenças autoimunes ou degenerativas. Há dois tipos principais de caspases, iniciadoras e efetoras. Caspases iniciadoras, como caspase- 8 e caspase-9, clivam pró-formas inativas de caspases efetoras, ativando-as. Caspases efetoras (caspase-3, caspase-7) por sua vez clivam outros substratos proteicos da célula resultando numa reação em cascata no processo apoptótico. ${ }^{79}$ Todas as caspases possuem como sítio ativo catalítico um resíduo de cisteína contendo o grupo tiol que pode ser oxidado por agentes alquilantes de grupos tióis, tais como iodoacetamida ou $\mathrm{N}$-etilmaleimida. $\mathrm{O}$ grupo funcional que caracteriza um ditiocarbamato possui habilidade tanto de complexação a centros metálicos diversos quanto de reagir frente a grupos tióis. Essas duas propriedades podem constituir em respostas dos possíveis efeitos biológicos exibidos por ditiocarbamatos.

Duas situações a princípio paradoxais são relatadas na literatura, enquanto ditiocarbamatos apresentam-se como inibidores potentes da apoptose, relata-se também sua capacidade de induzi-la, podendo atuar como pró-oxidantes e antioxidantes em diferentes situações. Ressalta-se que uma substância pró-oxidante pode induzir o processo de estresse oxidativo, resultando em danos a células e tecidos, ao passo que antioxidantes são capazes de inibir este processo. Dessa forma, ditiocarbamatos são capazes de causar morte celular ou inibir o processo de apoptose por diferentes estímulos. A ação anti-apoptótica é frequentemente associada ao seu efeito antioxidante, enquanto que a indução de apoptose correlaciona-se às suas propriedades oxidantes e quelantes frente a centros metálicos. Além disso, ambos os efeitos exibidos por ditiocarbamatos também têm sido relacionados à ativação ou inibição dos fatores NF-kB e AP-1. NF-kB e AP-1 (do inglês: nuclear factor kappa B e activator protein 1 , respectivamente) são fatores de transcrição que modulam a expressão gênica de diversos genes-chaves em processos apoptóticos, sendo ativados e estimulados por situação de estresse oxidativo. ${ }^{80} \mathrm{AP}-1$ é capaz de regular genes de neuropeptídeos e de enzimas envolvidas na biossíntese de neurotransmissores. NF-kB é um conjunto ou complexo de proteínas regulatórias ou fatores de transcrição envolvidos em processos celulares como respostas inflamatórias, imunológicas, de crescimento celular e apoptose, estando freqüentemente ativo no desenvolvimento de algumas doenças (câncer, doenças do coração e neuro-degenerativas, por exemplo). Na maioria das células, NF-kB está presente de forma inativa no citoplasma e mediante algum estímulo poderá ser ativado migrando para o núcleo celular. ${ }^{81}$

Alguns trabalhos importantes sobre a atuação dos DTCs em fenômenos apoptóticos foram publicados anteriormente na literatura ${ }^{82-85}$ Observou-se que o ditiocarbamato, (E)-2-oxo-2-[\{4(3-(3,4,5-trimetoxifenil)acriloil)fenil \}amino]etil-4-(2-hidroxietil) piperazino-1-DTC tem a propriedade de causar apoptose mitocondrial ao ativar essa enzima. ${ }^{86}$ Outro exemplo diz respeito ao ditiocarbamato contendo o grupo pirrolidina que inibiu a atividade de caspase- 3 em ratos com colite ulcerativa, induzida por tioacetamida. ${ }^{87}$ Outro estudo interessante revelou que complexos de $\mathrm{Au}(\mathrm{III})$ derivados de ditiocarbamatos contendo grupos bipiridina ativam as enzimas caspases-3 e -9, induzindo apoptose em algumas células de câncer resistente ao tratamento com cisplatina. ${ }^{88}$

A substância ziram, que é um ditiocarbamato derivado de $\mathrm{Zn}$ (II), Figura 12, tem a habilidade de induzir o processo de apoptose em células humanas através da ativação de caspases. ${ }^{89}$

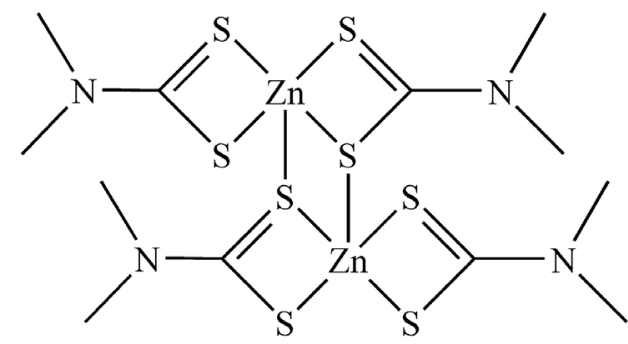

Figura 12. Representação estrutural do composto ziram

Experimentos com células epiteliais alveolares pulmonares expostas aos compostos pirrolidinoditiocarbamato, $\mathrm{CuCl}_{2}$ ou ao complexo de coordenação resultante da reação entre esses dois primeiros compostos, mostraram que o ligante e o cátion $\mathrm{Cu}$ (II) não afetam a função celular. Mas ao contrário deles, o $\mathrm{Cu}(\mathrm{DTC})_{2}$ diminuiu a viabilidade das células, induzindo mecanismo apoptótico ativando as caspases- $3,-7$ e $-9 .{ }^{90}$

\section{1ß-Hidroxiesteróide desidrogenases}

A ação e concentração de glicocorticoides em humanos são moduladas pelas enzimas ou isoenzimas 11-hidroxiesteróides dos tipos 1 e 2 (11-HSD1 e 11-HSD2). ${ }^{91}$ 11-HSD1 atua como uma enzima redutase, transformando cortisona em cortisol (uma forma inativa desse hormônio), enquanto 11-HSD2 age como uma enzima desidrogenase que catalisa o processo reverso. A 11-HSD1 contribui com o aumento de glucocorticóides no corpo, por exemplo cortisol, causando consequências clínicas como obesidade, principalmente visceral, hipertensão e osteoporose, sintomas da síndrome de Cushing. ${ }^{92}$ Já a enzima 11-HSD2 em humanos é importante na regulação eletrolítica dos rins, intestino e circulação sanguínea, e age na placenta como uma barreira de proteção fetal para uma alta concentração de cortisol.

Há duas décadas a literatura registrou os efeitos de inibição de ditiocarbamatos comerciais (Disulfiram, Thiram, dietilditiocarbamato (EDTC), difenilditiocarbamto (PDTC), [ $\left\{\mathrm{Zn}\left(\mathrm{S}_{2} \mathrm{CNCH}_{2} \mathrm{CH}_{2} \mathrm{NCS}_{2}\right)_{n}\right]$ (Zineb) e $\left[\left\{\mathrm{Mn}\left(\mathrm{S}_{2} \mathrm{CNCH}_{2} \mathrm{CH}_{2} \mathrm{NCS}_{2}\right)_{n}\right]\right.$ (Maneb) na enzima 11-HSD2. ${ }^{93}$ Um trabalho publicado em 2012 descreve a inibição da 
enzima 11 beta-hidroxiesteroide desidrogenase 2 (11 beta-HSD2) na presença da substância Thiram usando peixe zebra como modelo. ${ }^{94}$

\section{CONCLUSÕES}

Resumimos neste manuscrito os principais resultados de algumas pesquisas relatadas em artigos selecionados sobre a inibição de importantes enzimas por ditiocarbamatos (DTCs) e por alguns de seus complexos de coordenação. Apesar da importância das enzimas em diversos processos biológicos, vários relatos correlacionam suas atividades com o desenvolvimento de doenças como câncer, glaucoma, obesidade, hipertensão, osteoporose, dentre outras moléstias ou com o mecanismo de ação de microrganismos letais. Acredita-se que a glutationa, por exemplo, aumente a resistência ao tratamento do câncer usando drogas contendo Pt(II). Portanto, a inibição dessas enzimas por DTCs pode ter aplicações potenciais na medicina com implicações extremamente positivas. Acreditamos que este artigo pode ser importante para a química orgânica e inorgânica sintética, incentivando grupos de pesquisas interessados no assunto a preparar novos ligantes contendo ditiocarbamatos ou seus complexos de coordenação. Além disso, espera-se que pesquisadores em química medicinal tenham mais interesse em investigar as atividades biológicas dessas substâncias e possíveis mecanismos de ação baseados na inibição enzimática, contribuindo com o desenvolvimento dessa importante área de pesquisa e quem sabe gerando novas drogas para uso clínico.

\section{AGRADECIMENTOS}

Os autores agradecem aos seguintes órgão de financiamento de pesquisa: Conselho Nacional de Desenvolvimento Científico e Tecnológico - CNPq (304772/2017-8), Fundação de Amparo da Pesquisa do Estado de Minas Gerais - FAPEMIG (APQ-01626-14) e Coordenação de Aperfeiçoamento de Pessoal de Nível Superior Brasil - CAPES (99999.005687/2015-02).

\section{REFERÊNCIAS}

1. Morais, B. P.; de Lima, G. M.; Bitzer, R. S.; Donnici, C. L.; Wardell, J. L.; Rodrigues, B. L.; J. Organomet. Chem. 2017, 832, 57.

2. Morais, B. P.; de Lima, G. M.; Bitzer, R. S.; Donnici, C. L.; Wardell, J. L.; Rodrigues, B. L.; Polyhedron 2017, 135, 161.

3. Ferreira, I. P.;Wardell, J. L.; de Lima, G. M.; Wardell, S. M. S. V.; Paniago E. B., Pinheiro, C. B.; Inorg. Chim. Acta 2016, 441, 137.

4. Ferreira, I. P.; de Lima, G. M.; Paniago, E. B.; Takahashi, J. A.; Pinheiro, C. B.; J. Coord. Chem. 2014, 67, 1097.

5. Ferreira, I. P.; de Lima, G. M.; Pinheiro, C. B.; Ardisson, J. D.; Paniago, E. B.; Takahashi, J. A.; Rocha, W. R.; Polyhedron 2014, 79, 161.

6. Ferreira, I. P.; de Lima, G. M.; Pinheiro, C. B.; Ardisson, J. D.; Paniago, E. B.; Takahashi, J. A.; Inorg. Chim. Acta 2014, 443, 423.

7. Ferreira, I. P.; de Lima, G. M.; Pinheiro, C. B.; Paniago, E. B.; Krambrock, K.; Wardell, J. L.; Visentin, L. C.; J. Mol. Struct. 2013, 1048, 357.

8. Ferreira, I. P.; de Lima, G. M.; Pinheiro, C. B.; Ardisson, J. D.; Paniago, E. B.; Takahashi, J. A.; Rocha, W. R.; Eur. J. Med. Chem. 2012, 58, 493.

9. Menezes, D. C.; dos Santos, J. A. F.; de Lima, G. M.; Wardell, J. L.; Filgueiras, C. A. L.; Wardell, S. M. S. V.; Alcantara, A. F. de C.; Speziali, N. L.; J. Coord. Chem. 2012, 65, 559.

10. Cavalcanti, C. A.; Menezes, D. C.; de Lima, G. M.; dos Santos, J. A. F.; Ferreira, I. P.; Paniago, E. B.; Wardell, J. L.; Wardell, S. M. S. V.; Krambrock, K.; Mendes, I. C.; J. Mol. Struct. 2011, 988, 1.

11. Menezes, D. C.; de Lima, G. M.; Porto, A. O.; Coelho, M. G.; Augusti, R.; Ardisson, J. D.; Appl. Organomet. Chem. 2010, 24, 650.
12. Vale, J. A.; Faustino, W. N.; Menezes, P. H.; As, G. F.; J. Braz. Chem. Soc. 2006, 17, 829.

13. Kaur, M.; Mali, A. K.; Verma, N.; Singh, B.; Rao, A. L. J.; J. Braz. Chem. Soc. 2009, 20, 993.

14. Jardim, I. C. S. F.; Andrade, J. D.; de Queiroz, S. C. D.; Quim. Nova 2009, 32, 996.

15. Wang, X. J.; Xu, H. W.; Guo, L. L.; Zheng, J. X.; Xu, B., Guo, X.; Zheng, C. X.; Liu, H. M.; Bioorg. Med. Chem. Lett. 2011, 21, 3074.

16. Buac, D.; Schmitt, S.; Ventro, G.; Kona, F. R.; Dou, Q. P.; Mini Rev. Med. Chem. 2012, 12, 1193.

17. Adokoh, C. K.; RSC Adv.2020, 10, 2975.

18. Uchide, N.; Ohyama, K.; J. Antimicrob. Chemother. 2003, 52, 8.

19. Viola-Rhenals, M.; Patel, K. R.; Jaimes-Santamaria, L.; Wu, G.; Liu, J. Dou, Q. P.; Curr. Med. Chem. 2018, 25, 506.

20. Ramos, L. A.; Cavalheiro, E. T. G.; Chierice, G. O.; Quim. Nova 2008, 31,1714

21. Doadro, A. L.; Sotelo, J.; Fernandez-Ruano, A.; Quim. Nova 2002, 25, 525 .

22. Halimehjani, A. Z.; Ramezani, M.; Shamiri, E. V.; Hooshmand, S. E. Hashemi, M. M.; J. Braz. Chem. Soc. 2015, 26, 1500.

23. Vangaal, H. L. M.; Diesveld, J. W.; Pijpers, F. W. J.; Vanderlinden, G. M.; Inorg. Chem. 1979, 18, 3251.

24. Brinkhoff, H. C.; Cras, J. A.; Steggerda, J. J.; Willemse, J.; Recl. Trav. Chim. Pays-Bas 1969, 88, 633.

25. Andrew, F. P.; Ajibade, P. A.; J. Coord. Chem. 2019, 72, 1171.

26. Sachinidis, J.; Grant, M. W.; Aust. J. Chem. 1981, 34, 2195.

27. Cachapa, A.; Mederos, A.; Gili, P.; Hernández-Molina, R.; Domínguez, S.; Chinea, E.; Rodríguez, M. L.; Feliz, M.; Llusar, R.; Brito, F.; Galarreta, C. M. R.; Tarbraue, C.; Gallardo, G.; Polyhedron 2006, 25, 3366.

28. Warshawsky, A.; Rogachev, I.; Patil, Y.; Baszkin, A.; Weiner, L.; Gressel, J.; Langmuir 2001, 17, 5621.

29. Haryani, S.; Kurniawan, C.; Kasmui.; IOP Conf. Ser.: Mater. Sci. Eng. 2018, 349, 012060

30. de Paula da Silva, C. H. T.; Sanches S. M; Taft, C. A.; J. Mol. Graph. Modell. 2004, 23, 89.

31. Tomasello, M. F.; Nardon, C.; Lanza, V.; Di Natale, G.; Pettenuzzo, N.; Salmaso, S.; Milardi, D.; Caliceti, P.; Pappalardo, G.; Fregona, D.; Eur. J. Med. Chem. 2017, 138, 115.

32. Scintilla, S.; Brustolin, L.; Gambalunga, A.; Chiara, F.; Trevisan, A.; Nardon, C.; Fregona, D.; J. Inorg. Biochem. 2016, 165, 159.

33. Krishnamurthy, V. M.; Kaufman, G. K.; Urbach, A. R.; Gitlin, I.; Gudiksen, K. L.; Weibel, D. B.; Whitesides, G. M.; Chem. Rev. 2008, 108, 946.

34. Nocentini, A; Supuran, C. T.; Expert Opin. Drug Discovery 2019, 14, 1175.

35. Supuran, C. T.; Expert Opin. Drug Metab. Toxicol. 2020, 16, 197.

36. Vullo D.; Del Prete, S.; Nocentini A.; Osmand S. M.; AlOthmand Z.; Capasso C.; Bozdaga M; Carta F.; Gratterie P.; Supuran, C. T.; Bioorg. Med. Chem. 2017, 25, 1260.

37. Innocenti, A.; Scozzafava, A.; Supuran, C. T.; Bioorg. Med. Chem. Lett. 2009, 19, 1855 .

38. Carta, F.; Aggarwal, M.; Maresca, A.; Scozzafava, A.; McKenna, R.; Supuran, C. T.; Chem. Commun. 2012, 48, 1868.

39. Bozdag, M.; Carta, Vullo, Akdemir, A; Isik, S.; Lanzi, C.; Scozzafava, A.; Masini, E.; Supuran, C. T.; Bioorg. Med. Chem. Lett. 2015, 23, 2368.

40. Monti, S. M.; Maresca, A.; Viparelli, F.; Carta, F.; De Simone, G.; Mühlschlegel, F. A.; Scozzafava, A.; Supuran, C. T.; Bioorg. Med. Chem. Lett. 2012, 22, 859.

41. Aspatwara, A.; Hammaren, M.; Koskinena, S.; Luukinena, B.; Barkera, H.; Cartab, F.; Supuran, C. T.; Parikka M.; Parkkila, S.; J. Enzym. Inhib. Med. Chem. 2017, 32, 832. 
42. Wykoff, C. C.; Beasley, N. J.; Watson, P. H.; Turner, K. J.; Pastorek, J.; Sibtain, A.; Wilson, G. D.; Turley, H.; Talks, K. L.; Maxwell, P. H.; Pugh, C. W.; Ratcliffe, P. J.; Harris, A. L.; Cancer Res. 2000, 60, 7075.

43. Neri, D.; Supuran, C. T.; Nat. Rev. Drug Discovery 2011, 10, 767.

44. Liochev, S. I.; Fridovich, I.; Free Radical Bio. Med. 2007, 42, 1465.

45. Gressel, J. Synergizing herbicides.; Rev. Weed Sci. 1990, 5, 49.

46. Amstad, P.; Moret, R.; Cerutti, P.; J. Biol. Chem. 1994, 269, 1606.

47. Scott, M. D.; Meshnick, S. R.; Eaton, J. W.; J. Biol. Chem. 1987, 262, 3640 .

48. Yim, H. S.; Kang, J. H.; Chock, P. B.; Stadtman, E. R.; Yim, M. B. A.; J. Biol. Chem. 1997, 272, 8861.

49. Cao, R.; Fragoso A.; Villalonga, R.; Monatsh. Chem. 1996, 127, 775.

50. Díaz, A. M.; Villalonga, R.; Cao, R.; J. Coord. Chem. 2009, 62, 100.

51. Onwudiwe, D. C.; Ekennia, A. C.; Res. Chem. Intermed. 2017, 43, 1465.

52. Babo, S.; Vasseur, P.; Aquat. Toxicol. 1992, 22, 61.

53. Warshawsky, A.; Rogachev, I.; Patil, Y.; Baszkin, A.; Weiner, L.; Gressel, J.; Langmuir 2001, 17, 5621.

54. Krajewska, B. I.; J. Mol. Catal. B: Enzym. 2009, 59, 9.

55. Sumner, J. B.; J. Biol. Chem. 1926, 69, 435.

56. Balasubramanian, A.; Ponnuraj, K.; J. Mol. Biol. 2010, 400, 274.

57. Jabri, E.; Carr, M. B.; Hausinger, R. P.; Karplus, P. A.; Science 1995, $268,998$.

58. Krajewski, B.; Zaborska, W.; Bioorgan. Chem. 2007, 35, 355.

59. Kot, M.; Bicz, A.; J. Enzyme Inhib. Med. Chem. 2008, 23, 514.

60. Yamaguchi, K.; Hausinger, R. P.; Biochem. 1997, 36, 5118.

61. Fahey, J. W.; Stephenson, K. K.; Wade, K. L.; Talalay, P.; Biochem. Biophys. Res. Commun. 2013, 435, 1.

62. Menezes, D. C.; Borges, E.; Torres, M. F.; Braga, J. P.; Chem. Phys. Lett. 2012, 548, 85 .

63. Van Gelder, C. W. G.; Flurkey, W. H.; Wicbers, H. J.; Phytochemistry 1997, 45, 1309.

64. Amin, E.; Saboury, A. A.; Mansuri-Torshizi, H.; Zolghadri, S.; Iran. J. Chem. Eng. 2019, 38, 127.

65. Zolghadria, S.; Bahramia, A.; Khanb, M. T. H.; Munoz-Munozc, J.; Garcia-Molinad, F.; Garcia-Canovas, F.; Sabourye, A. A.; J. Enzym. Inhib. Med. Chem. 2019, 34, 289.

66. Townsend, D. M.; Tew, K. D.; Tapiero, H.; Biomed. Pharmacother. 2003, $57,145$.

67. Lin, T. H.; Rao, M. Y.; Lu, H. W.; Chiou, C. W.; Lin, S. T.; Chao, H. W.; Cheng, H. C.; Lee, T. M.; Physiol. Plant. 2018, 162, 35.

68. Narayanankutty, A.; Job, J. T.; Narayanankutty, V.; Curr. Prot. Pept. Sci. 2019, 20, 907.

69. Marozienè, A.; Lesanavičius, M.; Davioud-Charvet, E.; Aliverti, A.; Grellier, P.; Šarlauskas, J.; Čènas, N.; Molecules 2019, 24, 4509.

70. Elskens, M. T.; Penninckk, M. J.; J. Biochem. 1995, 231, 667.

71. Williams, M. R. M.; Bertrand, B.; Hughes, D. L.; Waller, Z. A. E.; Schmidt, C.; Ott, I.; O'Connell, M.; Searcey, M.; Bochmann, M.; Metallomics 2018, 10, 1655.
72. Boscutti, G.; Feltrin, L.; Lorenzon, D.; Sitran, S.; Aldinucci, D.; Ronconi, L.; Fregona, D.; Inorg. Chim. Acta 2012, 393, 304.

73. Ding, H.; Zhu, T.; Yin, X.; Liu, J.; Zhang, L.; Bernier, M.; Zhao, R.; Acta Biochim. Biophys. Sin. 2014, 46, 582.

74. Thompson, R. W.; Valentine, H. L.; Valentine, W. M.; Toxicol. Sci. 2002, $70,269$.

75. Kim, S. H.; Han, S. I.; Oh, S. Y.; Chung, H. Y.; Kim, H. D.; Kang, H. S.; Biochem. Biophys. Res. Commun. 2001, 281, 367.

76. Rahden-Staron, I.; Grosicka-Maciag E.; Kurpios-Piec D.; Czeczot H.; Grzela, T.; Szumiło, M.; Arch. Toxicol. 2012, 86, 1841.

77. Hosni, M.; Meskini, N.; Prigent, A. F.; Anker, G.; Joulain, C.; el Habib, R.; Lagarde, M.; Biochem. Pharmacol. 1992, 43, 1319.

78. Chu, G.; J. Biol. Chem. 1994, 269, 787.

79. Lamkanfi, M.; Festjens, N.; Declercq, W.; Berghe, T. V.; Vandenabeele, P.; Cell Death Differ. 2007, 14, 44.

80. Muller, J. M.; Rupec, R. A.; Baeuerle, P. A.; Methods Enzymol. 1997, 11,301 .

81. Sen, R.; Baltimore, D.; Cell 1986, 46, 705.

82. Stefan, C.; Nobel, I.; Kimland, M.; Nicholson, D. W.; Orrenius, S.; Slater, A. F. G.; Chem. Res. Toxicol. 1997, 10, 1319.

83. Bessho, R.; Matsubara, K.; Kubota, M.; Kuwakado, K.; Hirota, H.; Wakazono, Y.; Lin, Y. W.; Okuda, A.; Kawai, M.; Nishikomori, R.; Heike, T.; Biochem. Pharmacol. 1994, 48, 1883.

84. Pinkus, R.; Weiner, L. M.; Daniel, V.; J. Biol. Chem. 1996, 271, 13422.

85. Fernandez, P. C.; Machado, J. Jr.; Heussler, V. T.; Botteron, C.; Palmer, G. H.; Dobbelaere, D.; Biol. Chem. 1999, 380, 1383.

86. Fu, D. J.; Li, J. H.; Yang, J. J.; Li, P.; Zhang, Y. B.; Liu, S.; Li, Z. R.; Zhang, S. Y.; Bioorg. Chem. 2019, 86, 375.

87. Elmahmoudy, B. M.; El Fattah, M. A. A.; Elyamany, M. F.; Rashed, L. A.; Asian. Pac. J. Trop. Med. 2019, 9, 373.

88. Altaf, M.; Monim-U1-Mehboob, M.; Kawde, A. N.; Corona, G.; LArcher, R.; Ogasawara, M.; Celegato, M.; Borghese, C.; Siddik, Z. H.; Aldinucci, D.; Isab, A. A.; Oncotarget 2017, 8, 490.

89. Li, Q.; Kobayashi, M.; Kawada, T.; Int. J. Immunopathol. Pharmacol. 2012, 25,883 .

90. Chen, Y. W.; Chen, K. L.; Chen, C. H.; Wu; H. C., Su, C. C.; Wu, C. C.; Way, T. D.; Hung, D. Z.; Yen,;C. C.; Yang, Y. T.; Lu,T. H.; Toxicol. Lett. 2010, 199, 333.

91. Krozowski, Z.; Li, K. X. Z.; Koyama, K.; J. Steroid. Biochem. Mol. Biol. 1999, 69, 391.

92. Price, J. N.; Bertagna, X.; Grossman, A. B.; Lancet 2006, 367, 1605.

93. Niu, P.; Yang, K.; Biochim. Biophys. Acta 2002, 1594, 364.

94. Meyer, A.; Strajhar, P.; Murer, C.; da Cunha, T.; Odermatt, A.; Toxicology 2012, 301, 72 . 